University of Nebraska - Lincoln

DigitalCommons@University of Nebraska - Lincoln

Faculty Publications, Department of Physics and Astronomy

Research Papers in Physics and Astronomy

$9-2009$

\title{
The Aharonov-Bohm Effects: Variations on a Subtle Theme
}

Herman Batelaan

University of Nebraska - Lincoln, hbatelaan@unl.edu

Akira Tonomura

Hitachi, akira.tonomura.zk@hitachi.com

Follow this and additional works at: https://digitalcommons.unl.edu/physicsfacpub

Part of the Physics Commons

Batelaan, Herman and Tonomura, Akira, "The Aharonov-Bohm Effects: Variations on a Subtle Theme" (2009). Faculty Publications, Department of Physics and Astronomy. 113.

https://digitalcommons.unl.edu/physicsfacpub/113

This Article is brought to you for free and open access by the Research Papers in Physics and Astronomy at DigitalCommons@University of Nebraska - Lincoln. It has been accepted for inclusion in Faculty Publications, Department of Physics and Astronomy by an authorized administrator of DigitalCommons@University of Nebraska Lincoln. 


\title{
The Aharonov-Bohm effects: Variations on a subtle theme
}

\author{
Herman Batelaan and Akira Tonomura
}

The notion, introduced 50 years ago, that electrons could be affected by electromagnetic potentials without coming in contact with actual force fields was received with a skepticism that has spawned a flourishing of experimental tests and expansions of the original idea.

Herman Batelaan is a professor of physics at the University of Nebraska-Lincoln. Akira Tonomura is a fellow at Hitachi Ltd and a group director at the RIKEN Frontier Research System in Saitama, Japan. He is also a principal investigator at the Okinawa Institute of Science and Technology.

Quantum phenomena that were once thought to limit measurement capabilities are now being harnessed to enhance them and to improve the sensitivity of nanometer-size devices. Persistent questioning and probing of quantum phenomena has yielded many such advances. However, the Aharonov-Bohm effect, a modern cornerstone of quantum mechanics, is not yet a good example of that kind of progress. The AB effect was already implicit in the 1926 Schrödinger equation, but it would be another three decades before theorists Yakir Aharonov and David Bohm pointed it out. ${ }^{1}$ And to this day, the investigation and exploitation of the $A B$ effect remain far from finished.

Our discussion of the $\mathrm{AB}$ effect begins with a seemingly innocent question: What happens to an electron as it passes by an infinitely long ideal solenoid? One might expect that the elec-

Figure 1. Two electrons passing by a long current-carrying solenoid on opposite sides (along the blue trajectories) illustrate the magnetic Aharonov-Bohm effect. Although the solenoid's magnetic field (purple flux lines) is almost entirely restricted to the coil's interior, the vector potential A outside (tangential to the green coaxial circles) falls off only as $1 / r$ with distance $r$ from the axis. One of the two trajectories is parallel to $\mathbf{A}$ (at the point of closest approach), while the other is antiparallel. Even in the absence of any Lorentz force on the electrons, that difference produces a quantum mechanical phase shift between their wavefunctions. tron is unaffected. Outside the solenoid, the magnetic and electric fields B and E, and thus the Lorentz force, are all zero. But in quantum mechanics one describes an interaction not by the forces involved but rather by the Hamiltonian.

The canonical momentum in the electromagnetic Hamiltonian has a term proportional to the magnetic vector potential A, which is defined (with some gauge freedom) by $\mathbf{B}=\nabla \times \mathbf{A}$. Before the $\mathrm{AB}$ paper, the vector potential was generally thought of as a useful mathematical artifice without independent physical reality. Aharonov and Bohm changed all that.

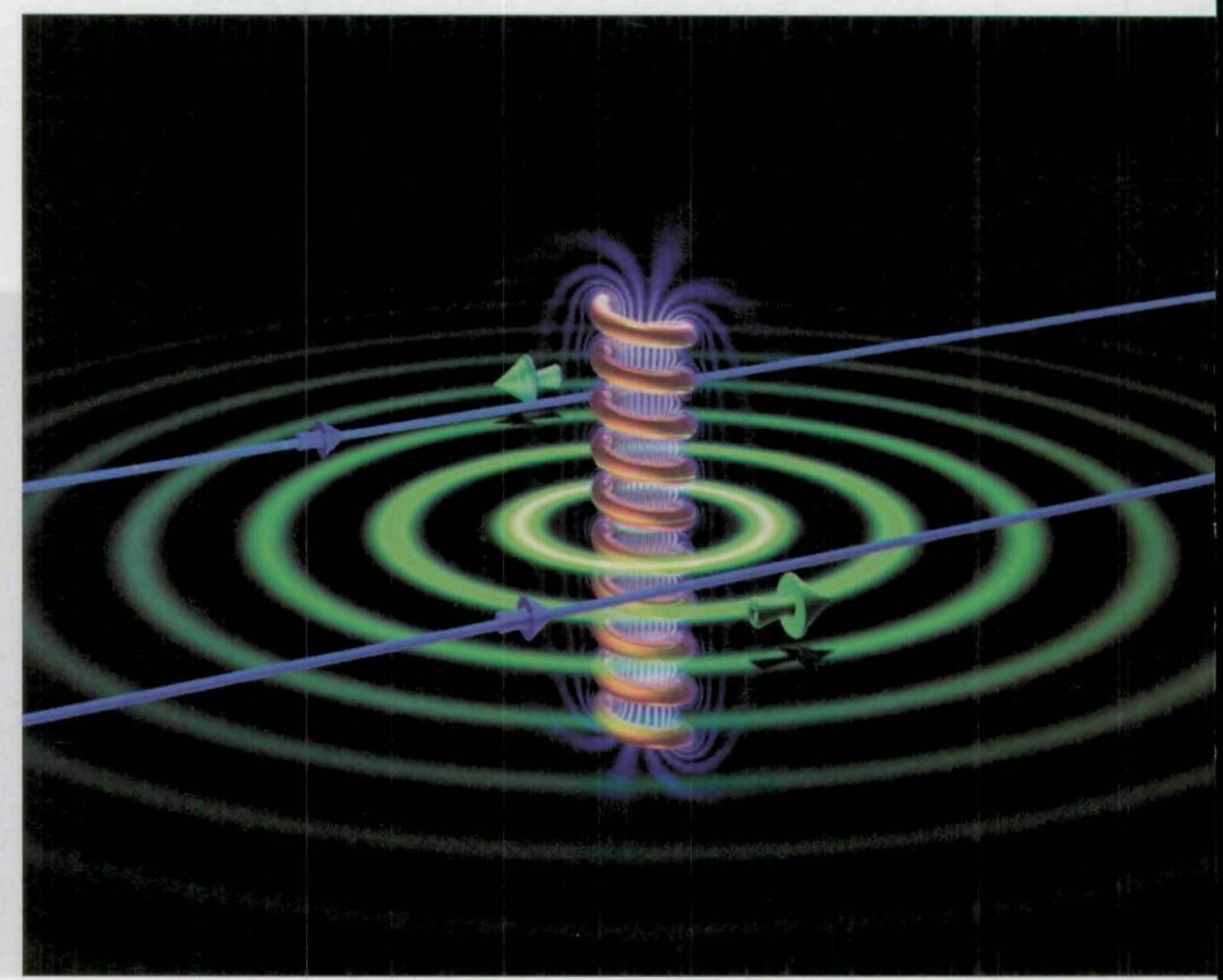


The first interesting fact is that $\mathbf{A}$ is not zero outside the solenoid, even though the force fields $\mathbf{E}$ and $\mathbf{B}$ vanish there (see figure 1). The vector potential is everywhere azimuthal to the solenoid's axis, and Stokes's theorem says that its line integral around any loop enclosing the solenoid equals the magnetic flux $\Phi$ inside. So outside the solenoid, A falls off like $1 / r$ with distance $r$ from the axis.

In figure 1, two electrons traveling together pass by the solenoid on opposite sides. At the points of closest approach to the solenoid, one is traveling parallel to $\mathrm{A}$ on its side, the other antiparallel. Quantum mechanically, one says that the electrons interact with the solenoid because the wavefunction of each accumulates a phase shift

$$
\varphi=-e / \hbar \int \mathbf{A} \cdot d \mathbf{l}
$$

as it traverses the vector potential.

The dot product in equation 1 implies that the phase changes are of opposite sign for electrons passing the solenoid on opposite sides. So the two electrons on their different paths past a long solenoid enclosing flux $\Phi$ accumulate a phase difference

$$
\Delta \varphi \equiv \varphi_{\mathrm{AB}}=(e / h) \Phi,
$$

known as the magnetic Aharonov-Bohm phase shift.

In 1959, beyond pointing out that quantum mechanics implied this phase shift in the absence of any classical force, Aharonov and Bohm suggested how it could be measured by having electron waves traversing the two paths interfere with each other. And so, indeed, was the $\mathrm{AB}$ phase shift soon confirmed in the laboratory. ${ }^{2}$ Achieving interference patterns requires that the initial electron beam be adequately coherent and that its splitting maintain that coherence. The former is accomplished by having a well-collimated beam with narrow spreads in angle and particle energy, and the latter requires something like a diffraction grating or an electromagnetic biprism. ${ }^{3}$

A heuristic example makes the existence of the $\mathrm{AB}$ phase shift less surprising and elucidates the classical-quantum correspondence. Figure 2 shows the constant-phase wavefront of a coherent electron beam passing through and around a magnetized bar with a homogeneous magnetic field. The electrons actually traversing the bar are deflected by the Lorentz force, and that part of the wavefront is correspondingly tilted. The wavefront for those parts of the beam that pass outside the bar remains untilted. But continuity requires the entire phase-constant wavefront to remain contiguous, so that one of its undeflected segments now lags the other, and a proper cross section of the beam shows a corresponding phase shift between electrons that passed undeflected on opposite sides of the bar.

Following through that simple semiclassical picture, one gets the same phase shift predicted for the system by the $\mathrm{AB}$ formalism. Note that the magnetized bar is circled, much like a solenoid, by an A field. The correspondence principle demands that in the classical limit, the effect of $\mathbf{A}$ on the electron wavefunction be the same as the deflection of a classical electron beam by the Lorentz force. From the correspondence argument, one might expect that $\mathrm{AB}$ phase shifts occur only in situations in which a charged-particle trajectory would be deflected. But the remarkable subtlety and curiosity of the effect is precisely that $\mathrm{AB}$ phase shifts occur in cases with no deflection.

\section{Scrutiny and skepsis}

Because of its nonobvious nature, the $\mathrm{AB}$ effect has endured considerable scrutiny. For example, the effect of stray fields

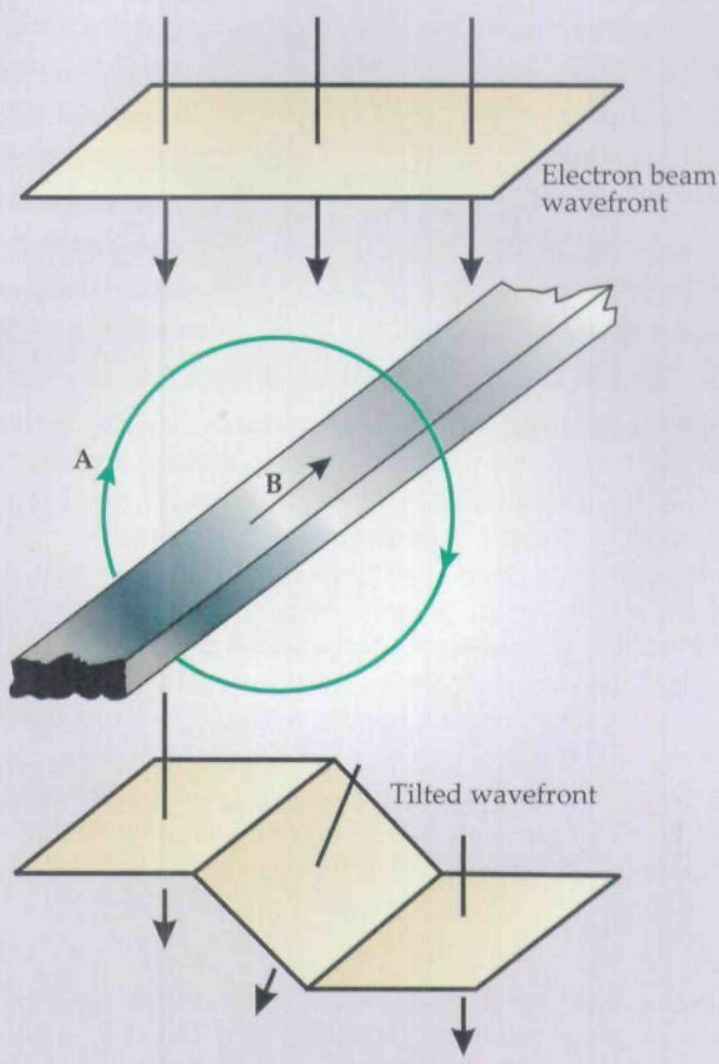

Figure 2. Semiclassical argument for the magnetic Aharonov-Bohm effect. Consider the plane wavefront of a coherent electron beam passing through and around a uniformly magnetized bar. Electrons going through the bar are deflected by the Lorentz force, tilting their sector of the wavefront. Electrons passing outside the bar see no magnetic field, so their sectors remain untilted. But because continuity requires the three sectors of the constant-phase front to remain contiguous, the two untilted sectors are now displaced along the beam direction, which implies a corresponding phase shift across a cross section of the beam. The phase shift thus calculated in terms of the Lorentz force is the same as that predicted by the $A B$ effect in terms of the vector potential $A$ circling the bar.

emanating from a finite solenoid was a concern in early attempts to test the effect. ${ }^{3}$ Could it be that the stray fields provide a force that makes the effect less purely quantum mechanical?

To visualize where such stray fields can be, consider the experimental scheme illustrated in figure 1 (and see the cover of this issue). An electron wavepacket is coherently split into two wavepackets that pass on opposite sides of the solenoid. Having thus acquired $\mathrm{AB}$ phase shifts of opposite sign, the two packets are recombined, and the resulting interference signal depends on the total magnetic flux enclosed by the two paths. It turns out that the measured phase shift agrees well with equation 2 , regardless of whether the experimenters use a solenoid or a magnetic whisker - a long sliver of magnetized metal. ${ }^{2}$ So it's unlikely that stray fields, which would depend on the details of the device providing the flux, could explain the phenomenon.

Another issue that arises with respect to stray fields is the unphysical character of the infinitely long solenoid generally invoked in discussions of the $\mathrm{AB}$ effect. So the $\mathrm{AB}$ effect 


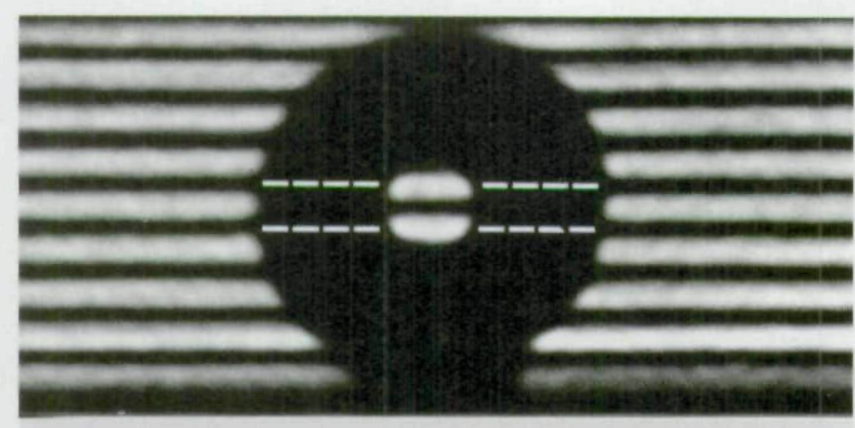

Figure 3. Electron interference pattern demonstrating the magnetic Aharonov-Bohm effect in an experiment that strictly excludes all stray fields. ${ }^{4} \mathrm{~A}$ coherent electron beam traveling normal to the page is made to pass around a toroidal magnet (seen as a shadow) or through its 4- $\mu$ mdiameter hole. The magnet's superconducting cladding prevents all stray fields. Having threaded or passed around the magnet, the beam is made to interfere with a reference plane wave. The resulting pattern, with the interference fringe inside the hole offset by half a cycle from those outside whenever the magnet flux is an odd multiple of $h / 2 e$, indicates an $A B$ phase shift of $\pi$ (modulo $2 \pi$ ) between the threading and bypassing electrons.

has sometimes been dismissed as an extreme limiting case based on an unphysical geometry. A 1986 experiment by a group at Hitachi led by one of us (Tonomura), done with a tiny toroidal magnet, is illuminating in that regard. ${ }^{4}$ The magnet's superconducting niobium cladding excluded any stray fields in that experiment. Its excellent agreement with the $A B$ prediction answers all objections about stray fields and unphysical geometries.

The electron interferogram in figure 3 manifests the $\mathrm{AB}$ effect demonstrated in the Hitachi experiment. A coherent electron beam is directed at a microfabricated toroidal magnet. Most of the beam passes by the outside of the magnet, but some of it threads through the magnet's 4- $\mu$ m-diameter central hole. A gold outer cladding prevents any electrons from penetrating the magnet itself, and the superconducting inner cladding keeps the hole and the entire vicinity of the magnet free of any stray B fields. But within the magnet's nonsuperconducting core, where no beam electrons venture, the circling $\Phi$ can be varied over several multiples of $h / e$. Equation 2 says that when $\Phi$ equals an odd multiple of the flux quantum $h / 2 e$, there should be an $\mathrm{AB}$ phase shift of $\pi$ (modulo $2 \pi$ ) between the electrons that threaded the hole and those that bypassed the magnet. And that's precisely what is seen in the interferogram that results from bringing the beam together with a coherent reference beam that avoided the magnet altogether.

\section{The electric $A B$ effect}

Given the many and varied confirmations of the magnetic $A B$ effect, it is no surprise that textbooks often present the AB effect as a beautiful and closed subject. Nevertheless, an increasing citation rate has pushed the total number of citations of the original $\mathrm{AB}$ paper beyond 2500, hardly what one expects for a closed subject with no applications in prospect. The continuing interest is generated, in part, by the fact that two versions of the $\mathrm{AB}$ effect were already described in the original 1959 paper: the magnetic version discussed above and the electric version.

If an enclosed magnetic flux can cause phase shifts, one might expect that an enclosed electric flux can do the same. And that is indeed what Aharonov and Bohm predicted. The electric analogue of equation 1 is a time integral of the electric scalar potential $V$ :

$$
\varphi=e / h \int V d t .
$$

That's plausible if one considers that $\mathbf{A}$ and $V$ make up a relativistic four-vector just like $\mathrm{x}$ and $t$.

The figure in box 1 compares the two original AB effects (as well as two others discussed below). Just as a split electron beam run past a solenoid magnet manifests the magnetic $A B$ effect (panel a), the two beams passed through separate charged metallic tubes demonstrate its electric analogue (panel b). There is essentially no electric field inside the tubes, and the electrons in the beam never experience the field between the tubes. Nonetheless, by maintaining a voltage difference between the tubes, one sees a measurable phase shift when the two beams subsequently interfere.

There are two versions of the electric $\mathrm{AB}$ experiment. In the steady-state version, the electrons do experience fringe electric fields as they enter and exit the tubes. But in the pulsed version, rapid charging and discharging of the tubes is done while the electron wavepackets are fully shielded inside them.

The steady-state version of the experiment was performed in 1985 by Giorgio Matteucci and Giulio Pozzi at the University of Bologna. ${ }^{5}$ The small size of electron interferometers prevented the use of actual metallic tubes. Instead, the experimenters ran the electrons past a bimetallic wire charged in such a way as to form a linear dipole field. In that configuration, the nonvanishing electric field produced no deflecting forces. The expected electric $\mathrm{AB}$ phase shift from passage on one side of the dipole field had the opposite sign of that on the other side. And that's what Matteucci and Pozzi found. At the time, their experiment was considered to be a demonstration of what is called a type II Aharonov-Bohm effect. The type I designation is reserved for experiments in which - in principle - the beam particles never traverse an $\mathbf{E}$ or B field (see box 1).

\section{Insidious mimics}

It is now understood, however, that something insidious occurs in the nonpulsed version of the electric $\mathrm{AB}$ experiment. Although the electrons are not deflected, they are delayed or sped up by Coulomb forces. When the electrons enter and exit the potentials, those Coulomb forces act in such a way as to mimic the expected phase shift. ${ }^{6}$ So the steady-state experiments do not really demonstrate the electric $\mathrm{AB}$ effect.

But can the pulsed versions entirely avoid those insidious forces? Perhaps there is also a force description that explains away the magnetic $\mathrm{AB}$ effect too. Maybe, pace Einstein, God is malicious as well as subtle. We could ask: Might the electron that passes by the solenoid be inducing changes in the solenoid that act back and impose on the electron a force that mimics the AB prediction ${ }^{6}$ (See box 2.)

To mimic the magnetic $\mathrm{AB}$ phase shift, the solenoid's reaction force would have to shift the electron wavepacket by a translation $\Delta x=\lambda \varphi_{\mathrm{AB}} / 2 \pi$, where $\lambda$ is the electron's de Broglie wavelength. For a wavepacket moving at velocity $v$, the resulting time delay would be $\Delta x / v$. Led by one of us (Batelaan), a group at the University of Nebraska has recently ruled out that reaction-force explanation with an experiment in which electron pulses were shot past solenoids and their arrival times were measured for different solenoid currents (see figure 4$){ }^{7}$ The experiment found no time delays that could mimic the magnetic $A B$ effect. 
The original magnetic and electric Aharonov-Bohm effects (panels $\mathrm{a}$ and $\mathrm{b}$ ) are type I effects in the sense that in an ideal experiment, the electron sees no $\mathbf{B}$ or $\mathbf{E}$ fields, though it does traverse different potentials $\mathbf{A}$ and $V$. In their respective dual effects - the Aharonov-Casher effect (panel c) and the so-called neutronscalar AB effect (panel d) - polarized neutrons (neutral particles with magnetic dipole moments) replace unpolarized electrons, and electrostatic configurations change places with solenoids. ${ }^{10}$ In panel c, a neutron interferometer encloses a line of charge, and in panel $\mathrm{d}$, neutrons pass through pulsed solenoids. These duals are classified as type II effects because the neutron must traverse a nonvanishing $\mathbf{E}$ or $\mathbf{B}$ field.

In either case, to acquire an $A B$ phase shift, the electron or neutron must pass through a region of nonzero electromagnetic potential. That quantum mechanical result seems to elevate the status of the potentials to a physical reality absent from classical electromagnetism. Yakir Aharonov has pointed out that the potentials do overdetermine the experimental outcome; the phase shift need only be known modulo $2 \pi$. An alternative view is that the original magnetic $A B$ effect shows electromagnetic fields acting nonlocally.

For type II effects, the wavepackets can plow straight through force fields, and forces are allowed in the interaction. But the $\mathrm{AB}$ interpretation requires
A loophole in the interpretation of the Nebraska experiment might be that its solenoids were much bigger than those that demonstrated the magnetic $\mathrm{AB}$ effect. ${ }^{6}$ Small solenoids might respond differently. It all depends on the interaction time versus the solenoid's response time. For electron velocities of about $10^{8} \mathrm{~m} / \mathrm{s}$ and a closest approach of about $10 \mu \mathrm{m}$ between the electron and a small solenoid, the shortest interaction time would be about $10^{-13}$ seconds. For the same beam velocity, a larger solenoid like those in the Nebraska experiment, with diameters on the order of millimeters, has interaction times a hundred times longer.

For comparison, the response time of electrons in a metal is typically $10^{-14}$ or $10^{-13}$ seconds. Thus the Nebraska delay experiment and the demonstrations of the magnetic $A B$ effect do allow the solenoids enough time to respond, albeit just barely for the small solenoids used in the demonstrations.

Perhaps the ultimate experiment is the one proposed in 1985 by Anton Zeilinger. ${ }^{8}$ So far in this discussion, we have only considered what a force does in the classical regime. Zeilinger considered what a force would do in the quantum regime. A large enough solenoid reaction force, he pointed out, would shift wavepackets so much in opposite directions that they would not overlap when recombined. That is to say, if solenoid reaction forces really were mimicking the predicted $A B$ effect, all interference contrast would disappear when the flux in the solenoid was big enough. But if the phase shift really is due to the $\mathrm{AB}$ effect, the interference contrast would remain unaffected beyond the length of the wavepacket-or, in a continous electron beam, the beam's coherence length $L_{\mathrm{c}}$. The coherence length of a continuous beam with a wavelength spread $\Delta \lambda$ is given by $\lambda^{2} / \Delta \lambda$. It's the separation along the beam beyond which any two points have lost all phase coherence.
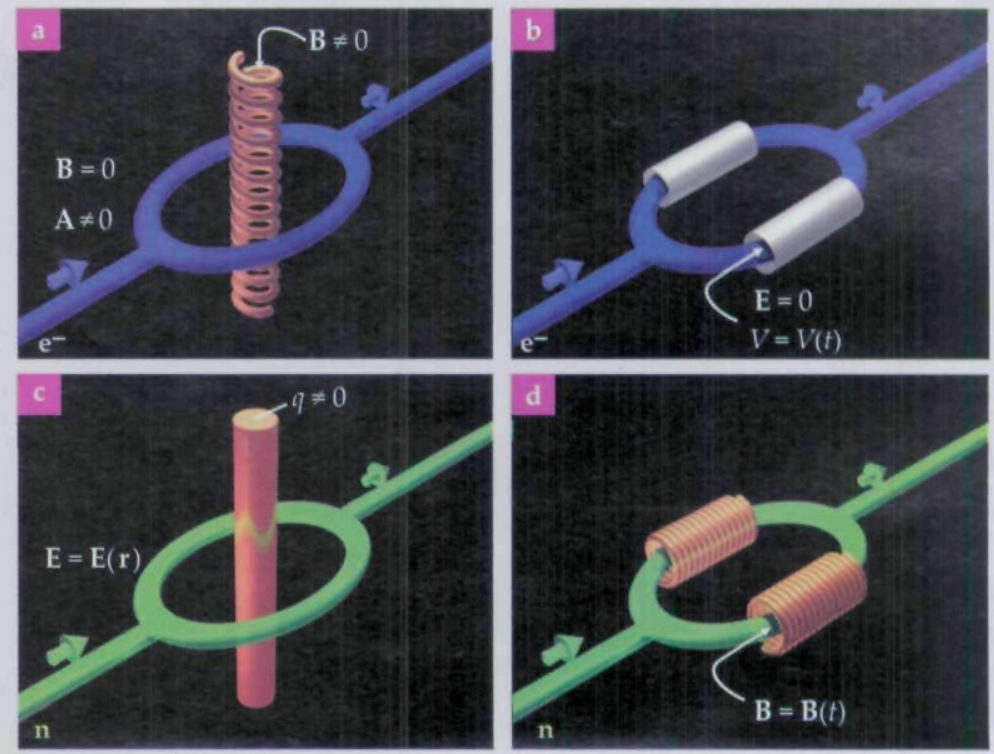

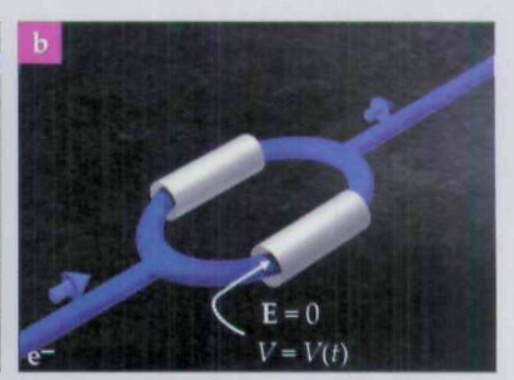

that the emerging wavepackets not be deflected or delayed in any way. Quantum mechanical descriptions generally circumvent the notion of forces. But one can use here an operational definition of forces that might be mimicking an $A B$ effect: If the interaction has produced no deflection or delay, there were no forces.

\section{Dispersion}

To visualize the shift of a wavepacket, as distinguished from a quantum phase shift, it is useful to describe what happens to the packet's frequency components. If each frequency component accumulates the same phase shift after passage by the solenoid-which is what the $A B$ effect predicts-the wavepacket's envelope function does not shift. That generally accepted view is captured by the concise statement that the $\mathrm{AB}$ effect is dispersionless. But if, on the other hand, some back-reaction force were giving different Fourier components different phase shifts that depended linearly on frequency, the wavepacket's envelope function would indeed shift. (See the interactive Flash movie accompanying the online version of this article.) Experimentally, the observation of electron fringes beyond the beam's coherence length would verify the $\mathrm{AB}$ effect's dispersionless character.

The extraordinary difficulty of such an experiment becomes clear when one compares the electrons' de Broglie wavelength to the coherence length, which is also the smallest possible wavepacket length for a given $\Delta \lambda$. In the relevant experimental regime, the typical $L_{\mathrm{c}}$ is $10^{5}$ times $\lambda$. A longer $\lambda$ means unacceptably low electron momentum, and a shorter $L_{c}$ means an unacceptably large momentum spread. So one would have to observe $10^{5}$ interference fringes to test for dispersion. Thus far such experiments, crucial as they are to the characterization of the $\mathrm{AB}$ effects, have remained out of reach.

Nor has the pulsed version of the original (type I) electric $\mathrm{AB}$ effect ever been performed. The development of new pulsed electron sources and detection schemes may change that situation. In 1999 Ahmed Zewail received the Nobel Prize in Chemistry for developing ultrafast sensing techniques (see PHYSICS TODAY, December 1999, page 19). Those 


\section{Box 2. A paradox}

Yakir Aharonov and Daniel Rohrlich have posed a fully classical paradox that they regard as "crucial for clarifying the entirely quantum interactions of fluxons [flux quanta] and charges-the generalized Aharonov-Bohm effect."17 The paradox involves the interaction of a charge with a magnetic flux tube, just as in the original magnetic $A B$ effect. The magnetic flux tube is realized with symmetric charge and current distributions (see the figure below). Two coaxial, counterrotating, and oppositely charged tubes create the flux. The difference between the two tube diameters is negligible; they rotate in opposite directions with the same rim speed $v$.

Outside the tubes there is no electric or magnetic field (the latter assuming the tubes are very long). So a charged particle at rest outside the tubes should stay where it is. But if friction between the tubes causes their spins to decelerate, something curious occurs. Faraday's law dictates that the diminishing magnetic flux inside the tubes induces an electric field outside. The electron experiences a force F and accelerates, but the tubes do not. What happened to momentum conservation?

Aharonov and Rohrlich reply that one must take account of momentum in the electromagnetic field and "hidden" relativistic mechanical momentum in the tubes. Many paradoxes are based on field momentum, and hidden momentum is a difficult concept that has been debated for decades. With regard to the $A B$ effect, identifying all the (relativistic) classical momentum terms and all the forces associated with the interaction between flux quanta and charges is not easy. ${ }^{18}$ So experiments have an important role to play.

techniques have until now been focused on probing femtosecond molecular dynamics. But one technique-ultrafast electron diffraction - also appears to be well suited for studying foundational issues of quantum mechanics such as the $\mathrm{AB}$ effect. That is especially true in view of the newest pulsedelectron sources such as those developed by Mark Kasevich and coworkers at Stanford University. ${ }^{9}$

\section{Duals}

Are the original magnetic and electric $\mathrm{AB}$ effects isolated phenomena? If they really exist, should they not be mirrored in other physical systems? Aharonov's answer is affirmative. In a 1984 paper, he and Aaron Casher predicted that both the magnetic and electric $\mathrm{AB}$ effects have their so-called duals, in which electric charges and magnetic dipoles exchange roles (see box 1 ). ${ }^{10}$ Just as the magnetic $\mathrm{AB}$ effect relies on the interaction of a moving charge with magnetic dipoles (box 1 , panel a), its dual (panel c) is found in the reversed situation: when a moving magnetic dipole-for example, a neutroninteracts with a line of charges (see PHYSICS TODAY, January 1990, page 17). The electric AB effect (panel b) has a dual in which neutrons pass through solenoids (panel d).

A series of experiments have confirmed those ideas. ${ }^{10}$ For example, in a tour de force 1992 experiment at the University of Melbourne, Brendan Allman and coworkers demonstrated the dual of the electric $\mathrm{AB}$ effect by passing longitudinally po-
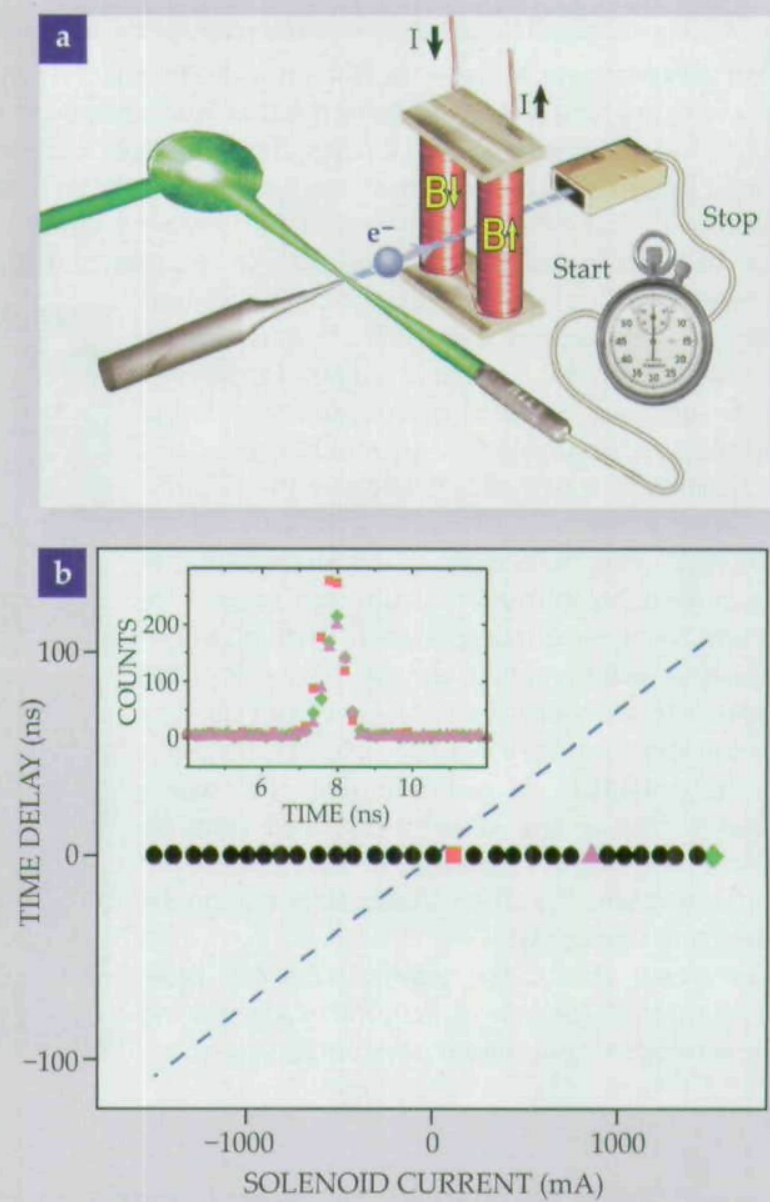

Figure 4. In search of forces that might mimic the magnetic Aharonov-Bohm effect, (a) electrons were sent through a field-free gap between solenoids, and (b) their flight times were measured as a function of the current flowing in opposite senses in the two solenoids. In the absence of current, the flight time between detectors is about $8 \mathrm{~ns}$. If a reaction force from the solenoids is to mimic the $A B$ effect, delays (or advances) relative to $8 \mathrm{~ns}$ would have to vary with current like the dashed diagonal line. Instead, the measured time delays are consistent with zero at all currents. The inset shows that the measured spectrum of flight times is essentially the same for low, high, and intermediate currents. (Adapted from ref. 7.)

larized neutrons through pulsed solenoids. They did find a phase shift that agreed with the prediction. But that dual is intrinsically a type II effect, because the neutrons must pass through a nonvanishing $\mathbf{B}$ field. Their polarization parallel to the solenoid field is meant to eliminate Lorentz forces. Nonetheless, the Melbourne group's interpretation was alternately disputed and supported by dueling theorists. The controversy was eventually resolved by a follow-up experiment that observed the phase shift beyond the neutron beam's coherence length, yielding a rare demonstration of the dispersionless character of an $\mathrm{AB}$ phenomenon. ${ }^{11}$

The dual of the magnetic AB effect is also a type II effect, in that the neutrons traverse regions of nonvanishing electric field. It was experimentally realized by enclosing charged, straight electrodes within the two arms of a neutron interferometer. ${ }^{12}$ It is interesting to compare that physical scenario 
with the Mott-Schwinger effect, in which neutron scattering off nuclei is attributed to the force between the nuclear charge and the incident neutron's magnetic dipole. ${ }^{13}$ On the other hand, the interactions of neutrons with line charges is considered to be a true $A B$ effect. As far as we know, there hasn't been a unified theoretical or experimental treatment of the two effects. With increasingly refined neutron sources becoming available, it may soon be possible to distinguish experimentally between the force-driven scattering of neutrons off a charged sphere and the presumed absence of such a force in their interaction with a charged line.

\section{Still more $A B$ phenomena}

Although solid-state manifestations of the $\mathrm{AB}$ effect are not the main topic of this article, we would hardly do justice to the breadth of the effect without mentioning them and the experimental breakthroughs that have made them possible. The first step was the 1985 observation, by Richard Webb and coworkers at IBM, of the $\mathrm{AB}$ effect for electrons circulating in a mesoscopic ring of nonsuperconducting metal ${ }^{14}$ (see PHYSICS TODAY, January 1986, page 17). Although the electrons were propagating within the metal, their interaction with the lattice did not cause enough decoherence to disrupt $\mathrm{AB}$ interference, even in the absence of superconductivity.

Not having to rely on superconductivity in the creation of a solid-state electron interferometer lets the experimenter avoid the complication of flux quantization, and it permits the investigation of new quantum effects in disordered materials. More recently, the observation of electron interference in carbon nanotubes suggests that the microscopic regime is also accessible for $\mathrm{AB}$ investigations. ${ }^{15}$

In all the solid-state experiments, mesoscopic and microscopic, the $A B$ phase shift was observed even though magnetic fields were present throughout the interferometer. That makes them a bit different from the enclosed-flux experiments with electron beams in vacuum. An important promise that those small solid-state electronic devices offer is their possible application in such areas as quantum computing.

Our discussion thus far has been based on interference between quantum mechanical amplitudes for alternative paths of a single particle. The physics of two-electron interference phenomena has to deal with the additional issue of Fermi-Dirac quantum statistics. A number of two-electron interference experiments in recent years have reported observing the predicted statistical phenomena of antibunching and Hanbury Brown-Twiss interference. ${ }^{16}$ (For background on HBT interference, see PHYSICS TODAY, August 2008, page 8.) The most recent of those experiments exhibited HBT interference that created orbital quantum entanglement between electron beams from two distinct sources.

Nowadays electrons can be used to demonstrate in the laboratory all the elements at the heart of quantum mechanics: wave-particle duality, quantum statistics, nonlocality, and entanglement. Because those elements are important to the prospects for quantum cryptography, quantum teleportation, and quantum computation, a new era of quantum electronics may be at hand.

Aharonov stresses that the arguments that led to the prediction of the various electromagnetic $\mathrm{AB}$ effects apply equally well to any other gauge-invariant quantum theory. In the standard model of particle physics, the strong and weak nuclear interactions are also described by gauge-invariant theories. So one may expect that particle-physics experimenters will be looking for new $\mathrm{AB}$ effects in new domains.

We thank Adam Caprez for the artwork. This article is based on work supported by the NSF under grant no. 0653182.

\section{References}

1. Y. Aharonov, D. Bohm, Phys. Rev. 115, 485 (1959).

2. R. G. Chambers, Phys. Rev. Lett. 5, 3 (1960); G. Möllenstedt, W. Bayh, Naturwissenschaften 49, 81 (1962).

3. A. Tonomura, Electron Holography, 2nd ed., Springer, New York (1999), pp. xi, 162; The Quantum World Unveiled by Electron Waves, World Scientific, Hackensack, NJ (1998).

4. A. Tonomura et al., Phys. Rev. Lett. 56, 792 (1986).

5. G. Matteucci, G. Pozzi, Phys. Rev. Lett. 54, 2469 (1985).

6. T. H. Boyer, Found. Phys. 32, 41 (2002).

7. A. Caprez, B. Barwick, H. Batelaan, Phys. Rev. Lett. 99, 210401 (2007).

8. A. Zeilinger, in Fundamental Aspects of Quantum Theory, V. Gorini, A. Figerio, eds., Plenum, New York (1985).

9. P. Hommelhoff et al., Phys. Rev. Lett. 96, 077401 (2006).

10. Y. Aharonov, A. Casher, Phys. Rev. Lett. 53, 319 (1984); B. E. Allman et al., Phys. Rev. Lett. 68, 2409 (1992); G. Badurek et al., Phys. Rev. Lett. 71, 307 (1993); W.-T. Lee et al., Phys. Rev. Lett. 80, 3165 (1998).

11. G. Badurek et al., Phys. Rev. Lett. 71, 307 (1993).

12. A. Cimmino et al., Phys. Rev. Lett. 63, 380 (1989).

13. J. Schwinger, Phys. Rev. 73, 407 (1948).

14. R. A. Webb et al., Phys. Rev. Lett. 54, 2696 (1985).

15. A. Bachtold et al., Nature 397, 673 (1999); J. Cao et al., Phys. Rev. Lett. 93, 216803 (2004).

16. M. Henny et al., Science 284, 296 (1999); W. D. Oliver et al., Science 284, 299 (1999); H. Kiesel, A. Renz, F. Hasselbach, Nature 418, 392 (2002); I. Neder et al., Nature 448, 333 (2007).

17. Y. Aharonov, D. Rohrlich, Quantum Paradoxes: Quantum Theory for the Perplexed, Wiley-VCH, Weinheim, Germany (2005), p. 177.

18. W. Shockley, R. P. James, Phys. Rev. Lett. 18, 876 (1967); H. A Haus, P. Penfield Jr, Phys. Lett. A 26, 412 (1968); W. H. Furry, Am. J. Phys. 37, 621 (1969); L. Vaidman, Am. J. Phys. 58, 978 (1990); T. H. Boyer, J. Phys. A 39, 3455 (2006). 